\title{
Placental Malaria and Pre-Eclampsia from the Lagos State University Teaching Hospital, Ikeja, Lagos Nigeria
}

\author{
Abidoye Gbadegesin'1, Sunday Soyemi², Joy Agbara' ${ }^{1}$, Adekunle Sobande ${ }^{1}$, Samuel Adebayo ${ }^{3}$ \\ ${ }^{1}$ Department of Obstetrics \& Gynaecology, Ikeja, Lagos, Nigeria \\ ${ }^{2}$ Lagos State University Teaching Hospital, Ikeja, Lagos, Nigeria \\ ${ }^{3}$ Department of Pathology and Forensic Medicine, College of Medicine, Lagos State University, Ikeja, Lagos, Nigeria \\ Email: gbade2000@yahoo.com
}

How to cite this paper: Gbadegesin, A., Soyemi, S., Agbara, J., Sobande, A. and Adebayo, S. (2019) Placental Malaria and Pre-Eclampsia from the Lagos State University Teaching Hospital, Ikeja, Lagos Nigeria. International Journal of Clinical Medicine, 10, 613-621.

https://doi.org/10.4236/ijcm.2019.1011050

Received: October 15, 2019

Accepted: November 12, 2019

Published: November 15, 2019

Copyright $\odot 2019$ by author(s) and Scientific Research Publishing Inc. This work is licensed under the Creative Commons Attribution International License (CC BY 4.0).

http://creativecommons.org/licenses/by/4.0/

\begin{abstract}
Objective: To determine the relationship between placental malaria infection and pre-eclampsia in a holo-endemic zone. Design: Prospective case-control study. Materials and Methods: One hundred and twenty seven (127) pregnant women with a diagnosis of pre-eclampsia in labour or having caesarean section served as cases while controls were 127 normotensive parturient women. They were recruited from the maternity unit of Ifako Ijaiye and Isolo General Hospitals, Lagos that served as secondary care centers. At delivery, either spontaneous vaginal delivery or by caesarean section, a $2.0 \mathrm{~cm} \times 2.0 \mathrm{~cm}$ placenta tissue was cut with scalpel and fixed in $10 \%$ formaldehyde in a specimen bottle and sent to the pathologist. Following this, $2.5 \mathrm{mls}$ of Cord blood and $2.5 \mathrm{mls}$ of the maternal venous blood were taken into separate EDTA bottles properly labeled at delivery, samples were sent to the haematology laboratory immediately for peripheral thick film smear for malaria parasite. Results were obtained from the laboratory and together with data from the case files, they were entered into SPSS version 16 for analysis. Independent Student t-test was used for significance for continuous variables while Chi-square was used for qualitative data. The significance was set at 0.05 . Results: There were statistically significant differences between the cases and controls regarding the maternal age, number of pregnancies, $p<0.05$ but none in the gestational age at delivery and birth weight, $p>0.05$. There was statistically significant difference between the two groups regarding the diagnosis of placental malaria as well as past history of alcohol intake and occupation, $p<0.05$. Binary logistic regression analysis showed that chronic placenta malaria infection was an independent risk factor for preeclampsia. Conclusion: Placental malaria infection was more common in patients with preec-
\end{abstract}


lampsia than their matched normotensive patients in our environment. At the same time, chronic malaria was found to be an independent risk factor for preeclampsia. More concerted efforts by all stake holders should be geared towards primary prevention together with early diagnosis and treatment of malaria especially in early pregnancy. This may reduce the incidence and complication of preeclampsia in our environment.

\section{Keywords}

Placental Malaria, Preeclampsia, Holoendemic Zone

\section{Introduction}

Malaria is a life-threatening parasitic disease transmitted by female anopheles mosquitoes. Today approximately $40 \%$ of the world's population mostly those living in the world's poorest countries are at risk of malaria [1]. Today malaria is found throughout the tropical and sub-tropical regions of the world and causes more than 300 million acute illnesses and at least one million deaths annually [1] [2]. Pregnant women and their unborn children are also particularly vulnerable to malaria, which is a major cause of perinatal mortality, low birth weight and maternal anaemia [2]. Although adult living in endemic areas acquires a protective immunity against developing severe malaria, women are susceptible to malaria pathogenesis when they become pregnant, especially in the first pregnancy. The clinical symptoms (syndrome) of what is termed maternal or Placental Malaria may include premature delivery, intrauterine growth restriction, stillbirth, abortions, maternal anaemia and death of the mother and the newborn [3] [4] [5]. The reason for the severe pathology associated with maternal malaria is the massive infestation of the placenta with Plasmodium falciparum Erythrocytes. The parasitized erythrocytes sequester in the placenta capillaries, which results in hypoxia, inflammatory reactions and chronic intervillositis.

While the placenta of infected women may be infested with parasitized erythrocytes, with parasite densities sometimes exceeding $50 \%$ of the total placenta erythrocyte count, the peripheral blood may remain free of parasites [3] [4]. This implies that the absence of peripheral malaria parasitaemia may not mean the absence of malaria Placenta infection. Sequestration of infected erythrocytes in the placenta is a virulence factor exclusively displayed by Plasmodium falciparum, and not observed for other human malaria parasites [6]. Malaria is the most frequent in first pregnancy [7], peaking between 13 and 16 weeks, and declining toward term.

Pre-eclampsia is another problem that is common in Sub-Saharan African and causes many deaths during pregnancy. It is defined as high blood pressure (hypertension) and protein loss in the urine. Preeclampsia is a disorder of widespread vascular endothelial malfunction and vasospasm with clinical manifestation after 20 weeks' gestation and can present as late as $4-6$ weeks postpartum. 
In developing nations, the incidence of the disease is reported to be $4 \%-18 \%$ [8] [9], with hypertensive disorders being the second most common obstetric cause of stillbirths and early neonatal deaths in these countries [10]. It is also a major cause of maternal mortality and morbidity. The causes of pre-eclampsia are unclear, but many factors are probably involved. Among the theories that have been proposed is that of the inflammation of the placenta that might play a part and that this effect may affect both the mother and the fetus. Seasonal changes in the incidence of pre-eclampsia have been described in tropics, which are consistent with malaria transmission periods [11].

While some of these studies have reported the association between malaria and pre-eclampsia and malaria and hypertension during pregnancy [12] [13], other studies failed to find a significant association between malaria and pre-eclampsia [14] [15]. Furthermore, some of these studies did not differentiate between pre-eclampsia and other types of hypertension.

In tropical countries, malaria and hypertension are common diseases of pregnancy. They have physiopathologic similarities such as placenta ischemia, endothelial dysfunction, and production of proinflammatory cytokines. Recent findings suggested their possible link [16].

Placental histology is considered the "gold standard" for malaria diagnosis in pregnancy for epidemiological or biological study purposes because it can show signs of active, chronic and past infections [17] [18].

However, due to limited technical expertise, such testing is rarely available in holoendemic areas like ours. Most studies in Sub-Saharan Africa have relied on the results of the placental smear, the sensitivity of which is low compared with the placental histopathology.

We have therefore conducted this study to assess the relationship between placental malaria infection and preeclampsia in our environment where the two conditions occur commonly.

\section{Materials and Methods}

\subsection{Study Site}

This is a case control prospective, study carried out at the Obstetrics and Gynaecology Department of Lagos State University Teaching Hospital (LASUTH), using its two Maternal and Child Care units of Isolo General Hospital, and Ifako-Ijaiye General Hospital, January to December 2017. Both serve as tertiary referral centres. They were either in labour or having Caesarean section.

Ethical clearance was obtained for this study from the ethical clearance committee of Lagos State University Teaching Hospital Ikeja. All participants were fully informed about the study. They were told clearly about their right to withdraw from the study for whatever reasons at any stage of the study without penalty.

\subsection{Sample Size Determination}

The sample size was determined by applying the formula for comparison of two 
proportions: [19]

$$
n=\frac{(u+v)^{2}\left\{p_{1}\left(100-p_{11}\right)+p_{2}\left(100-p_{2}\right)\right\}}{\left(p_{1}-p_{2}\right)^{2}}
$$

where $n=$ the desired minimum sample size for each group.

$u=$ One-sided percentage point of the normal distribution, corresponding to 1 -the power.

$V=$ Percentage point of the normal distribution, corresponding to the (two-sided) significance level.

$p_{1}=$ the estimated percentage of an attribute that is present in population 1 .

$p_{2}=$ the estimated percentage of an attribute that is present in population 2 .

At $95 \%$ confidence level, with $80 \%$ power, $v=1.96$ and $u=0.84$.

$p_{1}=19.6 \%$ and $p_{2}=7.7 \%$ (Percentage of pregnant women with Placental Malaria among those with pre-eclampsia and those without pre-eclampsia respectively in previous study) [20] [21].

$$
n=\frac{(0.84+1.96)^{2}\{19.6(100-19.6)+7.7(100-7.7)\}}{(19.6-7.7)^{2}}=126.59 \approx 127
$$

The calculate minimum sample size for each group was 127.

\subsection{Study Participants}

The study comprised of 127 asymptomatic pre-eclamptic patients and a control group of 127 asymptomatic normotensive patients. Some patients were excluded from the study once they denied consent, had history of fever in the last two weeks, currently on anti-malarial treatment, had a known medical disorder such as sickle cell anaemia or infection like Human Immunodeficiency virus. Pre-eclampsia was diagnosed if the patient had a systolic blood pressure of $\geq 140$ $\mathrm{mmHg}$ and or a diastolic blood pressure of $\geq 90 \mathrm{mmHg}$ with the presence of proteinuria $(\geq+1)$ at gestational age more than 20 weeks. Recruitment of participants was done consecutively for those that fulfill the criteria for inclusion in the study as preeclamptic while controls were those that were normotensive admitted for delivery about the same period and consent to the study.

\subsection{Histopathology}

At delivery, within 30 minutes, either spontaneous vaginal delivery or by caesarean section, a $2.0 \mathrm{~cm} \times 2.0 \mathrm{~cm} \times 2.0$ placenta tissue including the placental membrane was cut with scalpel and fixed in $10 \%$ buffered formaldehyde in a specimen bottle and sent to the pathologist. They were processed and embedded in paraffin wax and sectioned onto slides by standard techniques. The placental biopsy samples were then processed and were embedded in paraffin wax. In every case, paraffin sections $4 \mathrm{~mm}$ thick were stained with hematoxylin-eosin and Giemsa stain. Because the samples were fixed in buffered formalin, formalin pigment formation, which has similar optical characteristics and polarized light activity to malaria pigment was not detected. Placental malaria infections were 
characterized based on the classification of Bulmer et al. [22]; uninfected (no parasites or pigment), acute (parasites in intervillous spaces), chronic (parasites in maternal erythrocytes and pigment in fibrin or cells within fibrin and/or chorionic villous syncytiotrophoblast or stroma), past (no parasites and pigment confined to fibrin or cells within fibrin.

\subsection{Laboratory Procedure}

$2.5 \mathrm{mls}$ of the maternal venous blood was taken into separate EDTA bottles properly labelled at delivery, samples were sent to the haematology laboratory immediately for peripheral thick film smear for malaria parasite. In case of delivery in the night, the samples were preserved in the refrigerator till the following morning when they were sent to the laboratory. Microscopic examination of blood smears was done under oil immersion for parasite detection and 200 high power fields were used for the examination before the smear is considered negative. Parasites were counted against 200 leucocytes assuming an average leucocyte count of 8000 per microlitre of blood.

The data obtained were analysed using SPSS, version 17.0; a statistical computer program. Independent Students T-test was used to analyze continuous variables while Chi-square was used for categorical data. Binary Logistic Regression was used to test the independent risk factor for preeclampsia. The level of significance was set at 0.05 .

\section{Results}

The demographic and other continuous characteristics are shown in Table 1. There were statistically significant differences between the two groups regarding maternal age, gravidity, $p<0.05$ but not in birth weight, $p>0.05$. There was no significant difference with regards to history of smoking between the two groups, $p>0.05$. Table 2 showed that there were statistically significant differences between the two groups with regards to histological diagnosis of acute malarial infection and chronic infection, $p<0.05$, but not in past infection, $p>$ 0.05. Parasites and haemozoin pigments were seen in $35(27 \%)$ in the pre-eclampsia group while it was seen in $2(1.5 \%)$ of the normotensive group with a p-value $<0.05$. This was statistically significant. Similarly, active parasites were seen in 9 pre-eclamptic patients while absence in the control group. This was also statistically significant $(p<0.05)$.

Binary logistic regression showed that only chronic placental malaria infection was an independent risk factor for placental malaria, $p<0.05$ (Table 3 ).

\section{Discussion}

The major findings of this study were that placental malaria infection was significantly associated with and was an independent risk factor for preeclampsia in holoendemic region for malaria like ours. We also showed that chronic and active malaria infections were significantly more in patients with preeclampsia $(27.5 \%$ vs. $1.5 \%$ and $7 \%$ vs. $0.0 \%$ ) respectively. These values are higher than those 
Table 1. Demographic and other characteristics between the two groups.

\begin{tabular}{cccc}
\hline Characteristic Mean \pm SD & Group 1 & Group 2 & Significance \\
\hline N $=127$ & $31.25 \pm 5.79$ & $33.02 \pm 6.33$ & $p<0.05^{*}$ \\
Maternal age (years) & $2.05 \pm 1.29$ & $2.89 \pm 1.42$ & $p<0.05^{*}$ \\
Gravidity & $1.50 \pm 0.57$ & $1.33 \pm 0.51$ & $p>0.05$ \\
Miscarriages & $39.38 \pm 1.25$ & $39.28 \pm 1.42$ & $p>0.05$ \\
Gestational age at delivery (weeks) & $3175 \pm 0.54$ & $3288 \pm 0.41$ & $p>0.05$ \\
Birth weight (grams) & & & \\
\hline Level of education & $8(6.6)$ & $0(0)$ & $p<0.05^{*}$ \\
None & $28(21.7)$ & $11(8.8)$ & \\
$1^{\circ}$ & $64(50)$ & $45(35.4)$ & $p<0.05^{*}$ \\
$2^{\circ}$ & $28(21.7)$ & $71(33.8)$ & $p>0.05$ \\
$3^{\circ}$ & $3(2.3)$ & $12(9.4)$ & $p<0.05^{*}$ \\
Consumed alcohol & $6(4.9)$ & $12(9.8)$ & \\
Ever smoked & $60(47.5)$ & $3(2.4)$ & \\
\hline Maternal venous blood positive for & & & \\
malaria parasites & & &
\end{tabular}

$\mathrm{SD}=$ Standard deviation, ${ }^{*}=$ significant statistically.

Table 2. Histopathological diagnosis of placental malaria between the two groups.

\begin{tabular}{cccc}
\hline Characteristic.n (\%) & $\begin{array}{c}\text { Group 1 } \\
\text { N = 127 }\end{array}$ & $\begin{array}{c}\text { Group 2 } \\
\text { N = 127 }\end{array}$ & Significance \\
\hline Acute infection (active) & $9(7.0)$ & $0(0)$ & $p<0.05^{*}$ \\
Haemozin (past) & $50(39.3)$ & $50(39.3)$ & $p>0.05$ \\
Thickening of basement membrane & $13(10.2)$ & $35(27.5)$ & $p<0.05)^{\star}$ \\
Calcification & $69(54.3)$ & $64(50.3)$ & $p<0.05^{*}$ \\
Prominent synchytial knots & $94(74)$ & $92(72.4)$ & $p>0.05$ \\
Parasites/pigments and fibolin (chronic) & $35(27.5)$ & $2(1.5)$ & $p<0.05^{*}$ \\
\hline
\end{tabular}

* = significant statistically.

Table 3. Logistic regression identifying the risk factors for preeclampsia.

\begin{tabular}{ccccccc}
\hline & B & SE & Wald & df & Sig & Exp (B) \\
\hline Age & 0.028 & 0.022 & 1.600 & 1 & 0.206 & 1.029 \\
Ever consumed alcohol & -1.030 & 0.665 & 2.398 & 1 & 0.121 & 0.357 \\
Acute malaria infection & 6.247 & 4.462 & 0.000 & 1 & 0.999 & 516.37 \\
${ }^{*}$ Chronic malaria infection & 1.060 & 0.276 & 14.752 & 1 & 0.000 & 2.887 \\
Constant & -27.174 & 1.785 & 0.000 & 1 & 0.999 & 0.000 \\
\hline
\end{tabular}

* = significant statistically.

of Adam et al. [16] from an area of unstable malaria transmission in Sudan. However, the rates of past infection between the two groups (39.3\%) were similar. This is plausible, given the environment from where the study was underta- 
ken. The results of Sartlet et al. [12] are in concordance with ours. Nonetheless, some studies found an association between placental malaria infection and gestational hypertension, but not preeclampsia or eclampsia [13]. It is possible for placental malaria to be associated with preeclampsia given the similar pathophysiology of the two entities namely; placental ischemia, endothelial dysfunction and production of inflammatory cytokines.

Our study also revealed that more patients with preeclampsia had significantly higher malaria parasites in the maternal venous and cord blood than normotensive controls. The implication is that these may be used as a possible screening for placental malaria infection. However, placental parasitaemia without peripheral parasitaemia may occur in women who have previously been treated with clearance of peripheral parasites. On the other hand, peripheral parasitemia without placental infection may occur in early malaria infection, especially if parasitemia is low. Nonetheless, other studies found no correlation between placental and peripheral venous parasitemia [20].

We also found in our study that some socio-demographics like young age, primiparity, low level of education were more common in patients with preeclampsia. These are very well established associations [21]. Interestingly, our result may not support the concept that smoking is negatively associated with preeclampsia.

The limitations of this study were that the patients were not matched and the fact that we did not find out whether the patients used insecticide treated nets. We also do not know how long these patients have been living in Nigeria. There could be a possibility that some of them have not been in malarial endemic region for years and subsequently could not have developed the malaria immunity expected in holo-endemic region.

\section{Conclusion}

In conclusion, chronic placental malaria infection was shown to be an independent risk factor for preeclampsia in our environment with stable malaria transmission. We, therefore, advocate that more efforts at prevention through health information dissemination should be intensified and at the same time, prompt diagnosis and treatment of malaria, especially in the first half of pregnancy should be institutionalized.

\section{Conflicts of Interest}

The authors declare no conflicts of interest regarding the publication of this paper.

\section{References}

[1] World Health Organization (2010) Roll Back Malaria. WHO Report.

[2] Opare-Addo, O.A.T. (2001) Malaria in Pregnancy. Comprehensive Obstetrics in the Tropics, 34, 250-260.

[3] Mendez, C. (1995) Malaria during Pregnancy: A Priority Area of Malaria Research 
and Control. Parasitology Today, 11, 178-183.

https://doi.org/10.1016/0169-4758(95)80151-0

[4] Ismail, M.R., Ordi, J., Menendex, et al. (2000) Placenta Pathology in Malaria: A Histological, Immunohistochemical and Quantitative Study. Human Pathology, 31, 85-93. https://doi.org/10.1016/S0046-8177(00)80203-8

[5] Menendez, C., Ordi, J., Ismail, M.R., Ventura, P.J., Aponte, J.J., Kahigwa, L., Font, F., et al. (2000) The Impact of Placental Malaria on Gestational Age and Birth Weight. The Journal of Infectious Diseases, 181, 1740-1745.

https://doi.org/10.1086/315449

[6] Andrews, K.T. and Lanzer, M. (2002) Maternal Malaria: Plasmodium falciparum Sequestration in the Placenta. Parasitology Research, 88, 715-723.

https://doi.org/10.1007/s00436-002-0624-5

[7] Walker-Abbey, A., Djokam, R.T., Eno, A., Leke, R.G., Titanji, V.P.K., Fogako, J., et al. (2005) Malaria in Pregnant Cameroonian Women: The Effect of Age and Gravidity on Submicroscopic and Mixed-Species Infection and Multiple Parasite Genotypes. The American Journal of Tropical Medicine and Hygiene, 72, 229-233. https://doi.org/10.4269/ajtmh.2005.72.229

[8] Villar, J., Betram, A.P. and Gulmezoglu, M. (2001) Epldemiological Basis for the Planning of Maternal Health Services. WHO/RHR.

[9] Khedum, S.M., Moodley, J., Naicher, T., et al. (1997) Drug Management of Hypertensive Disorders of Pregnancy. Pharmacology \& Therapeutics, 74, 221-258. https://doi.org/10.1016/S0163-7258(97)82005-0

[10] Ngoc, N.T., Meriaidi, M., Abdel-Aleem, H., et al. (2006) Causes of Stilbirths and Early Neonatal Deaths: Data from 7993 Pregnancies in Six Developing Countries. Bulletin of the World Health Organization, 84, 699-705. https://doi.org/10.2471/BLT.05.027300

[11] Wacker, J., Schullz, M., Fruhauf, J., Chiwora, F.M., Solomayer, E. and Bastert, G. (1998) Seasonal Change in the Incidence of Pre-Eclampsia in Zimbabwe. Acta Obstetricia et Gynecologica Scandinavica, 77, 712-716. https://doi.org/10.1080/j.1600-0412.1998.770703.x

[12] Sartelet, H., Rogier, C., Milko-Sartelet, I., Angel, G. and Michel, C. (1996) Malaria Associated Pre-Eclampsia in Senegal. The Lancet, 347, 1121. https://doi.org/10.1016/S0140-6736(96)90321-9

[13] Ndao, C.T., Dumont, A., Fievet, N., Doucoure, S., Gaye, A. and Lehesran, J.Y. (2009) Placental Malarial Infection as a Risk Factor for Hypertensive Disorders during Pregnancy in Africa: A Case-Control Study in an Urban Area of Senegal, West Africa. American Journal of Epidemiology, 170, 847-853. https://doi.org/10.1093/aje/kwp207

[14] Shulman, C.E., Marshall, T., Dorman, E.K., Bulmer, J.N., Cutts, F., Peshu, N., et al. (2001) Malaria in Pregnancy: Adverse Effects on Haemoglobin Levels and Birthweights in Primigravidae and Multigravidae. Tropical Medicine \& International Health, 6, 770-778. https://doi.org/10.1046/j.1365-3156.2001.00786.x

[15] Dorman, E.K., Shulman, C.E., Kingdom, J., Bulmer, J.N., Mwendwa, J., Peshu, N., et al. (2002) Impaired Uteroplacental Blood Flow in Pregnancies Complicated by Falciparum Malaria. Ultrasound in Obstetrics \& Gynecology, 19, 165-170. https://doi.org/10.1046/j.0960-7692.2001.00545.x

[16] Adam, I., Elhassan, E.M., Mohmmed, A.A., Salih, M.M. and Elbashir, M.I. (2011) Malaria and Pre-Eclampsis in an Area with Unstable Malaria Transmission in Central Sudan. Malaria Journal, 10, Article No. 258. 
https://doi.org/10.1186/1475-2875-10-258

[17] Bruce-Chawatt, L.J. (1925) Malaria in Infants and Children in Southern Nigeria. Annals of Tropical Medicine and Parasitology, 46, 173-200. https://doi.org/10.1080/00034983.1952.11685522

[18] Bulmerj, N., Resheed, F.N., Morrison, L., Francis, N. and Green wood, B.M. (1993) Placental Malaria: A Semi-Quantitative Investigation of the Pathological Features. Histopathology, 22, 219-225. https://doi.org/10.1111/j.1365-2559.1993.tb00111.x

[19] Varkevisser, C.M., Pathmanathan, I. and Brownlee, A. (1991) Designing and Conducting Health Systems Research Projects. International Development Centre, Ottawa and WHO, Geneva, Vol. 2, 216.

[20] Matelli, A., Caligaris, S., Casltelli, F. and Carosi, G. (1997) The Placenta and Malaria. Annals of Tropical Medicine and Parasitology, 91, 803-810. https://doi.org/10.1080/00034983.1997.11813206

[21] Agomo, C.O., Oyibo, W.A., Anorlu, R.I. and Agomo, U.A. (2009) Prevalence of Malaria in Pregnant Women in Lagos in Lagos, South-West Nigeria. The Korean Journal of Parasitology, 47, 179-183. https://doi.org/10.3347/kjp.2009.47.2.179

[22] Bulmer, J.N., Rasheed, F.N., Francis, N., Morrison, L. and Greenwood, B.M. (1993) Placental Malaria-Pathological Classification. Histopathology, 22, 211-218.

https://doi.org/10.1111/j.1365-2559.1993.tb00110.x 